\title{
The Role of Body Weight and Growing in Body Height to Nonspecific Musculoskeletal Pain in a Cohort of Bosnia and Herzegovina Schoolchildren
}

\section{A concise and informative title: Musculoskeletal pain during grow in height}

\author{
Nurka Pranjic $^{1,2}$, Selma Azabagic ${ }^{3}$ \\ 1 Department of Occupational Health, Faculty of Medicine, University of Tuzla, Univerzitetska 1, 75000 Tuzla, \\ Bosnia and Herzegovina \\ 2 Departments for Occupational Pathology and Toxicology, Health Care Centre in Tuzla, Albina Herljevica 1, \\ 75000 Tuzla Bosnia and Herzegovina \\ 3 Institute of Public Health in Tuzla Canton, 75 000Tuzla, Tuzlanskog odreda 6, 75000 Tuzla, Bosnia and \\ Herzegovina
}

\section{Correspondence to:}

Prof. Nurka Pranjic, MD, PhD

Faculty of Medicine, University of Tuzla,

Univerzitetska 1, 75000 Tuzla, Bosnia and Herzegovina,

Phone: +38735320600

E-mail: pranicnurka@hotmail.com

E-mail: nurka.pranjic@untz.ba

\begin{abstract}
Background Children often suffer the nonspecific musculosceletal pain as reported in literature. Aim To determine relationship between body weights with development of musculoskeletal pain and to determine whether growing in body height is associated with musculoskeletal pain in schoolchildren.

Subjects/ Methods A prospective longitudinal study included 1315 school children aged 7-14 years (652 boys and 663 girls) and was performed in 13 elementary schools in B\&H. Child body height and body weight were measured. The survey of perception of musculoskeletal pain in different body regions of subjects was conducted by adjusted Nordic Musculosceletal Questionnaire (NMQ).
\end{abstract}


Results The highest prevalence of an overweight and obesity in the $10^{\text {th }}$ year $35.7 \%$ and the lowest frequency $17.8 \%$ in the $14^{\text {th }}$ year was. In the age $14^{\text {th }}$ obesity was'nt found. Boys have more prevalence of overweight. Using logistic regression model, we found that school children with normal BMI were protected with increased body height of acute lower back pain $(\beta=-0.089,95 \% \mathrm{CI},-9.730--0.023, \mathrm{P}<0.049)$, and increased body height was protector of obese school children of acute upper back pain $(\beta=-0.356,95 \% \mathrm{CI},-14.077--3.878, \mathrm{P}<0.001)$ and chronic lower back pain $(\beta=-0.356,95 \% \mathrm{CI},-14.077--3.878, \mathrm{P}<0.001)$.

Conclusion Schoolchildren with normal weight more often have had musculosceletal pain than those with overweight or obesity. This can be associated with intense physical growth period in height, especially. The assumption is that the increase in height changes the relationship between excessive BMI and musculoskeletal pain in children of school age.

Key words: nonspecific musculoskeletal pain, body height, body weight, schoolchildren

\section{Introduction}

A musculoskeletal pain (MSP) in children is important public health problem, which is manifested in only occasional limiting movements with pain and usually with little clinical implications [1]. A nonspecific musculoskeletal pain (NSMSP) in childhood includes often a specific, identifiable pathoanatomical basis for symptoms cannot be found, resulting in non-specific diagnoses based on the location of symptoms [2]. Amongst various risk factors, it has been suggested that elevated body mass index (BMI), overweight and obesity might be an independent risk factor for NSMSP [3]. The relationship between BMI and MSP has mainly been investigated in studies on low back pain (LBP) [4-6]. Body height was associated with increased risk of low back pain in both genders [7]. MSP in the lower extremities occurs both in children and in adolescents with ankle and foot problems being more common in children [8]. There is emerging evidence that children, especially adolescents who report persistent musculoskeletal pain, are at increased risk of chronic pain as adults $[1,2,5]$. The time of adolescence is the transition period from childhood to adulthood and over only a few years, both body and soul will undergo many changes. The most apparent morphological differences are increased height and a change in body composition. It has been proposed that these may impact LBP [8-10].

The lack of research into relation of MSP with overweight and obesity in children means that understanding of these conditions is limited and health care professionals have little empirical evidence to underpin their clinical practice [10-11]. We examined the association between body mass index and MSP in different body regions and association between growing in body height and MSP in different body regions in school children aged from 9 to 14 years. 


\section{Subjects and Methods}

A prospective longitudinal study was performed from September 2016 to January 2017 in elementary schools in Tuzla Canton, in each of 13 municipality's institutions. Tuzla Canton as most populated Bosnia and Herzegovina canton has about 500000 citizens, out of which 24027 are children age 9 to 14 years. This research data containing elements that could lead to identification of respondents: the number of sex participants, some anthropometric measures data (body weight and body height, body mass index) including year of birth (age) as indirect identifiers of human research participants as indirect sensitive data. Data collection was performed by means of an anonymous and voluntary participation of all respondents in a part of questionnaire surveys and in a part of data collection of anthropometric measurements of body height and body weight which were taken by trained clinical staff. Written informed consent was obtained from parents for each child, respondents in a study. This study was approved by written authorization from the Ministry of Education, Science, Culture and Sport of Tuzla Canton and head of each participating school. The ethical approval for this research was obtained from the appropriate research committee at the Tuzla University School of Medicine (No: 03-8373-8.3.5/16.).

We analyse non-specific musculoskeletal pain in different body regions. We do not discuss pain associated with congenital or systemic diseases, such as hip dysplasia, juvenile arthritis or scoliosis. We also exclude pain resulting from frank injuries e.g. anterior ligament rupture, ankle sprain, fractures and pain following surgical interventions.

\section{Study population}

The study population included 2500 children who attended 3 th to 7 th grade $(10.4 \%$ children of total their population). Respondents who did not answer all of the questionnaire items or who refused to take part in the survey were excluded. The total sample of study included 1315 out of 2500 children, response rate was $54 \%$. There were no statistical differences of the respondents by gender, 652 (49.58\%) boys and 663 (50.41\%) girls. We visited, randomly selected, one primary school institution in every municipalities where we randomly selected 2500 children for our study.

We used cluster sample method in forming a representative sample of respondents: random selection of 2-5 respondents of one class repeated over 1 lecture (average of daily number of lectures 4-5), and 20 school children was selected from each school class ( $\geq 100$ respondents per one elementary school institution). Excluding factor for study participation was the existence of congenital or acquired deformities and physical disorders (children, who use wheelchairs, with child musculoskeletal disease, determined disparity between the lower extremities, problems with the foot etc.). 
Interview

The survey was conducted by specially designed questionnaire. The questionnaire consisted of two parts: the first part consisted of data on demographic and individual characteristics of participants and the second part was The Nordic Musculosceletal Questionnaire (NMQ) [12], which was translated to Bosnian language by team of two experts. NMQ quantifies musculoskeletal pain in 9 body regions: neck, shoulders, upper back, elbows, low back, wrist/hands, hips/thighs, knees and ankles/feet [12-13]. The internal consistency of each 16 items used about acute and chronic various body region NMQ questionnaire items in our study sample was excellent (Cronbach' alpha $=0.859)$.

\section{Anthropometric measurement}

Child anthropometric measurements were taken by trained clinical staff. Weight was measured using GIMA scale (model 27310 Astra) to the nearest of $0.001 \mathrm{~kg}$ and height was measured using SECA 213 portable stadiometer. For reliability, all measurements were taken in the duplicates and average. The scale is calibrated before each measurement. The obtained values of body weight and body height were used to calculate body mass index (BMI) as a ratio of body weight by the square of height. BMI were calculated and were expressed in percentiles also, to determine the nutritional status of school children due to changes in BMI by age and gender [14].

\section{Body mass index}

BMI groups were classified according to US Centre for Disease Control (CDC) and Prevention's age- and sexmatched percentile grading: underweight $(<5 \%)$, normal, healthy weight $(5 \%<85 \%)$, overweight $(85 \%<95 \%)$ and obese $(\geq 95 \%)^{(18)}$; and z-score. The risk of developing obesity has children, whose BMI are above the 85 and 95 percentiles for age [14].

\section{Statistical Analysis}

We performed data analysis using IBM SPSS Statistics for Windows, Version 19.0, IBM Corp., and Armonk, NY. The descriptive statistics are presented by means and standard deviations, or relative numbers and percentages for categorical data. To examine differences between- and within groups of acute and chronic pain according NMQ different body regions (neck pain, right shoulder, right wrist, upper back, lower back, hips, knees, ankles); and BMI categories groups we used ANOVA, sum of squares test. Non-parametric Spearman correlation test was used to analyse correlation relationships between NMQ scales and body height as NMQ scales and body weight; NMQ and Spearman's correlations were computed to evaluate relationships between mean item scale scores on each of the eight factors of the NMQ and body weight. Logistic regression analysis ANOVA were used to provide multivariate association ( predictive or protective potential) between NMQ scales (independent variables) and body height among schoolchildren who are divided in four BMI category as selected variables (body weight as dependent variables). All p-values $<0.05$ were regarded as statistically significant. 


\section{Results}

Between individual characteristics of respondents the mean by standard deviation (SD) were for: age $11.31 \pm 1.48$ years; sex 1.50 \pm 0.50 ; school class $5.05 \pm 1.42$; body height $146 \pm 10.61$; body weight $41.57 \pm 11.71$; BMI mass index $60.89 \pm 30.83$ percentiles, and MSP $1.47 \pm 0.49$. The higher average values of musculoskeletal pain regarding of

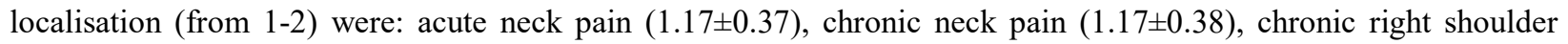

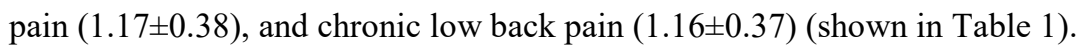

Table 1 Individual characteristics of respondents with perception of musculosceletal pain $(n=1315)$

\begin{tabular}{|l|c|c|c|}
\hline Characteristics of subjects & Mean \pm SD* & Minimum & Maximum \\
\hline Age (years) & $11.31 \pm 1.48$ & 7.00 & 14.00 \\
\hline Sex & $1.50 \pm 0.50$ & 1.00 & 2.00 \\
\hline School class & $5.05 \pm 1.42$ & 3.00 & 7.00 \\
\hline Body Weight $(\mathrm{kg})$ & $41.57 \pm 11.71$ & 20.00 & 93.00 \\
\hline Body Height $(\mathrm{cm})$ & $146.18 \pm 10.61$ & 113.50 & 180.00 \\
\hline Body mass index- BMI (m ${ }^{2}$ & $19.17 \pm 3.7$ & 9.84 & 40.25 \\
\hline Body mass index- BMI (percentile) & $60.89 \pm 30.83$ & 4.00 & 96.00 \\
\hline BMI categories & $2.39 \pm 0.77$ & 1.00 & 4.00 \\
\hline Musculosceletal Pain & $1.47 \pm 0.49$ & 1.00 & 2.00 \\
\hline Acute neck pain & $1.17 \pm 0.37$ & 1.00 & 2.00 \\
\hline Chronic neck pain & $1.17 \pm 0.38$ & 1.00 & 2.00 \\
\hline Acute right shoulder pain & $1.03 \pm 0.18$ & 1.00 & 2.00 \\
\hline Chronic right shoulder pain & $1.17 \pm 0.38$ & 1.00 & 2.00 \\
\hline Acute right wrist pain & $1.01 \pm 0.09$ & 1.00 & 2.00 \\
\hline Chronic right wrist pain & $1.01 \pm 0.07$ & 1.00 & 2.00 \\
\hline Acute upper back pain & $1.10 \pm 0.31$ & 1.00 & 2.00 \\
\hline Chronic upper back pain & $1.12 \pm 0.33$ & 1.00 & 2.00 \\
\hline Acute low back pain & $1.10 \pm 0.30$ & 1.00 & 2.00 \\
\hline Chronic low back pain & $1.16 \pm 0.37$ & 1.00 & 2.00 \\
\hline Acute hip pain & $1.03 \pm 0.18$ & 1.00 & 2.00 \\
\hline Chronic hip pain & $1.04 \pm 0.19$ & 1.00 & 2.00 \\
\hline Acute knees pain & $1.05 \pm 0.22$ & 1.00 & 2.00 \\
\hline Chronic knees pain & $1.06 \pm 0.25$ & 1.00 & 2.00 \\
\hline Acute ankles pain & $1.06 \pm 0.24$ & 1.00 & 2.00 \\
\hline Chronic ankles pain & $1.06 \pm 0.23$ & 1.00 & 2.00 \\
\hline SD, Standard Devian & & & \\
\hline
\end{tabular}

*SD, Standard Deviation

Table 2 shows the differences between each individual characteristics and perception of MSP of all respondents per BMI categories. We found the highest prevalence of an overweight BMI and obesity in the $10^{\text {th }}$ year being $35.7 \%$ (in the $11^{\text {th }}$ was $31.3 \%$; in the $12^{\text {th }}, 32.5 \%$; and in the $13^{\text {th }}, 30.8 \%$ ). The lowest frequency of excessive body weight was $17.8 \%$ in the age group of 14 , when obesity was not observed. On the other hand, an underweight BMI category was 
most frequent in the 14th year, 8.3\%, and is not negligible in the 10 th year, $6.8 \%$ also. An underweight was highest in the $14^{\text {th }}$ year, $8.3 \%$, and is not negligible in the $10^{\text {th }}$ year, $6.8 \%$. This can be associated with intense physical growth period in height, especially. In the ninth year, excessive BMI weight also had a high prevalence of $27.4 \%$. It was interesting to analyze the frequency of obesity by age. The highest frequency of obesity is in the 9th year (18.5\%), followed by a decreasing trend (in the tenth of $15.3 \%$, in the eleventh $14.4 \%$, in the twelfth $11.8 \%$ in the thirteenth $8.7 \%$, and in the fourteenth $0 \%$ ). There are statistically significant differences in BMI categories by age of life ( $\mathrm{P}<0.003$, data not presented). The frequency of underweight MBI among girls is almost two times higher than the frequency of underweight MBI among boys (41:23 vs. 6.2\%: 3.5\%). Boys have more prevalence of overweight (127: 116 vs. $19.5 \%$ : 17.5\%). Obesity is more than twice as frequent in boys as it is in girls $114: 52$ vs. $17.5 \%$ : $7.2 \%$. BMI by gender is significantly different ( $\mathrm{P}<0.001$, data not presented). When comparing the BMI category of respondents regarding the school classes, excess body weight is most common in the sixth grade $20.8 \%$ and in fourth grade $20.6 \%$. Obesity had the highest prevalence in the third class $19.5 \%$, and has obvious declining trend in the next classes. As far as the appearance of musculoskeletal pain and the activity of our subjects are concerned, there are no significant statistical differences in the appearance of musculoskeletal pain between those who exercise sport and those who do not. When we compare MSP and BMI categories we discover the inversion. Pain is increasing in the category of underweight (33: 31 vs. 5.3\%: 4.5\%), although declining in subjects with overweight (110: 133 vs. $17.6 \%: 19.2)$ and in the category of obese (65: 101 versus $10.4 \%: 14.6 \%)$.

School children have perceived chronic pain in neck $221(16.8 \%)$, upper back 158 (12\%), and low back 214 $(16.3 \%)$. The most common chronic musculosceletal pain was suffered by respondents with normal (healthy) body weight, overweight and obesity BMI categories. The prevalence rate of acute musculoskeletal pain was: right shoulder $10.2 \%$, neck 17\%, upper back $10.5 \%$ and low back $10.2 \%$ of the examinee. The most frequently acute musculosceletal pain is located in the right shoulder, chest and back. Underweight respondents did not perceived acute neck pain $(0 \%)$ (Table 2$)$. 
Table 2 The differences between individual characteristics and perception of MSP of all respondents per BMI categories $(n=1315)$

\begin{tabular}{|c|c|c|c|c|c|}
\hline Characteristics & $\begin{array}{c}\text { Underweight } \\
64(4.9 \%)\end{array}$ & $\begin{array}{c}\text { Normal } 842 \\
(64.0 \%)\end{array}$ & $\begin{array}{l}\text { Overweight } \\
243(18.5 \%)\end{array}$ & $\begin{array}{c}\text { Obesity } \\
166(12.6 \%)\end{array}$ & $\begin{array}{c}\chi^{2 *} \\
\text { P-value }\end{array}$ \\
\hline \multicolumn{6}{|l|}{ Age (years) } \\
\hline 9 & $8(4.8)$ & $114(67.8)$ & $15(8.9)$ & $31(18.5)$ & \multirow{6}{*}{$\begin{array}{c}26.959 \\
0.029\end{array}$} \\
\hline 10 & $19(6.8)$ & $161(57.5)$ & $57(20.4)$ & $43(15.3)$ & \\
\hline 11 & $5(1.8)$ & $182(66.9)$ & $46(16.9)$ & $39(14.4)$ & \\
\hline 12 & $11(4.5)$ & $155(63.0)$ & $51(20.7)$ & $29(11.8)$ & \\
\hline 13 & $15(5.5)$ & $176(63.7)$ & $61(22.1)$ & $24(8.7)$ & \\
\hline 14 & $6(8.3)$ & $54(73.9)$ & $13(17.8)$ & $0(0.0)$ & \\
\hline \multicolumn{6}{|l|}{ Gender } \\
\hline Male & $23(3.5)$ & $388(59.5)$ & $127(19.5)$ & $114(17.5)$ & \multirow{2}{*}{$\begin{array}{c}14.528 \\
0.002\end{array}$} \\
\hline Female & $41(6.2)$ & $454(68.5)$ & $116(17.5)$ & $52(7.8)$ & \\
\hline \multicolumn{6}{|l|}{ School class } \\
\hline $3 \mathrm{rd}$ & $13(5.3)$ & $164(65.3)$ & $25(9.9)$ & $49(19.5)$ & \multirow{4}{*}{$\begin{array}{l}11.658 \\
0.473\end{array}$} \\
\hline 4th & $12(4.7)$ & $152(59.8)$ & $52(20.6)$ & $38(14.9)$ & \\
\hline 5 th & $11(4.1)$ & $177(65.3)$ & $53(19.5)$ & $30(11.1)$ & \\
\hline 6th & $12(4.7)$ & $161(63.1)$ & $53(20.8)$ & $29(11.4)$ & \\
\hline \multicolumn{6}{|l|}{ Play sport } \\
\hline no & $34(5.7)$ & $393(64.6)$ & $112(18.4)$ & $69(11.3)$ & \multirow{2}{*}{$\begin{array}{l}0.985 \\
0.151\end{array}$} \\
\hline yes & $30(4.3)$ & $449(63.5)$ & $131(18.5)$ & $97(13.7)$ & \\
\hline \multicolumn{6}{|l|}{ MS- pain } \\
\hline no & $31(4.5)$ & $426(61.7)$ & $133(19.2)$ & $101(14.6)$ & \multirow{2}{*}{$\begin{array}{l}8.678 \\
0.034\end{array}$} \\
\hline yes & $33(5.3)$ & $416(66.7)$ & $110(17.6)$ & $65(10.4)$ & \\
\hline $\begin{array}{l}\text { Musculoskeletal pain, acute } \\
\text { or chronic in different part } \\
\text { of the body }\end{array}$ & Underweight & Normal & Overweight & Obesity & $\chi^{2}$, P-value \\
\hline Acute neck pain & $0(0.0)$ & $148(11.3)$ & $41(3.1)$ & $21(1.6)$ & $2.727,0.436$ \\
\hline Chronic neck pain & $12(0.9)$ & $133(10.1)$ & $49(3.7)$ & $27(2.1)$ & $2.001,0.572$ \\
\hline Acute right shoulder pain & $17(1.3)$ & $176(13.4)$ & $38(2.9)$ & $16(1.2)$ & $10.736,0.013$ \\
\hline Chronic right shoulder pain & $0(0.0)$ & $28(2.1)$ & $9(0.7)$ & $5(0.4)$ & $2.213,0.529$ \\
\hline Acute right wrist pain & $2(1.2)$ & $17(1.3)$ & $6(0.5)$ & $2(1.2)$ & $2.363,0.501$ \\
\hline Chronic right wrist pain & $3(0.2)$ & $20(1.5)$ & $2(0.2)$ & $2(0.2)$ & $5.415,0.144$ \\
\hline Acute upper back pain & $8(0.6)$ & $96(7.3)$ & $19(1.4)$ & $15(1.0)$ & $2.859,0.414$ \\
\hline Chronic upper back pain & $8(0.6)$ & $109(8.3)$ & $20(1.5)$ & $21(1.6)$ & $6.222,0.101$ \\
\hline Acute low back pain & $9(0.7)$ & $91(6.9)$ & $21(1.6)$ & $13(1.0)$ & $1.468,0.690$ \\
\hline Chronic low back pain & $12(0.9)$ & $138(10.5)$ & $34(2.6)$ & $30(2.3)$ & $4.076,0.253$ \\
\hline Acute hips pain & $2(0.2)$ & $30(2.3)$ & $7(0.5)$ & $6(0.5)$ & $0.704,0.872$ \\
\hline Chronic hips pain & $4(0.3)$ & $33(2.5)$ & $6(0.5)$ & $7(0.5)$ & $1.257,0.739$ \\
\hline Acute knees pain & $3(0.2)$ & $42(3.2)$ & $15(1.2)$ & $8(0.6)$ & $2.542,0.468$ \\
\hline Chronic knees pain & $3(0.2)$ & $53(4.0)$ & $18(1.4)$ & $10(0.8)$ & $2.007,0.557$ \\
\hline Acute ankles pain & $4(0.3)$ & $52(4.0)$ & $17(1.3)$ & $7(0.5)$ & $2.038,0.564$ \\
\hline Chronic ankles pain & $3(0.2)$ & $51(3.9)$ & $15(1.1)$ & $9(0.7)$ & $2.165,0.564$ \\
\hline
\end{tabular}

${ }^{*} \chi^{2}-$ Chi square test 
There are highly significantly positive correlations (at the level of $\mathrm{P}<0.001$ ) between body height and acute MSP of neck, right shoulder, upper back, low back, hips, knees, ankles and significantly positive correlation between body height and right wrist pain. Increased body height correlated with all acute MSPs in different body parts. Unexpected, increased body height was correlated with chronic MSPs in right wrist, upper back, hips, knees, ankles, neck pain and right shoulder. We did not found correlation between body height and chronic low back pains. Body weight correlated highly positive only with acute knees pains. There are body weight correlations (at the level of $\mathrm{P}<$ 0.05) with acute pains in neck, upper back, lower back, hips and ankles. Overweight and obese highly correlated with chronic knees pains in school children. On other site, body weight was correlated with chronic MSPs in right shoulder, upper back and ankles pains (shown in Table 3).

Table 3 Correlation between body height and MSP and body weight and MSP in different part of the body in all respondents $(n=1315)$

\begin{tabular}{|c|c|c|c|}
\hline $\begin{array}{l}\text { Spearman correlation between body } \\
\text { height }(113-173 \mathrm{~cm}) \text { and MSP }\end{array}$ & $\begin{array}{l}\text { Correlation } \\
\text { factor }\end{array}$ & $\begin{array}{l}\text { Spearman correlation between body } \\
\text { weight }(20.0-36.8 \mathrm{~kg}) \text { and MSP }\end{array}$ & $\begin{array}{l}\text { Correlation } \\
\text { factor }\end{array}$ \\
\hline Chronic neck pain & $0.068 * *$ & Chronic neck pain & 0.053 \\
\hline Chronic right shoulder pain & $0.080 * *$ & Chronic right shoulder pain & $0.084 * *$ \\
\hline Chronic right wrist pain & $0.095^{*}$ & Chronic right wrist pain & 0.033 \\
\hline Chronic upper back pain & $0.098 *$ & Chronic upper back pain & $0.061 * *$ \\
\hline Chronic lower back pain & 0.041 & Chronic lower back pain & 0.024 \\
\hline Chronic hips pain & $0.012 *$ & Chronic hips pain & 0.020 \\
\hline Chronic knees pain & $0.127 *$ & Chronic knees pain & $0.097 *$ \\
\hline Chronic ankles pain & $0.113^{*}$ & Chronic ankles pain & $0.087 * *$ \\
\hline Acute neck pain & $0.117 *$ & Acute neck pain & $0.069 * *$ \\
\hline Acute right shoulder pain & $0.138^{*}$ & Acute right shoulder pain & 0.053 \\
\hline Acute right wrist pain & $0.087 * *$ & Acute right wrist pain & 0.023 \\
\hline Acute upper back pain & $0.125 *$ & Acute upper back pain & $0.063 * *$ \\
\hline Acute lower back pain & $0.147^{*}$ & Acute lower back pain & $0.072 * *$ \\
\hline Acute hips pain & $0.112 *$ & Acute hips pain & $0.073 * *$ \\
\hline Acute knees pain & $0.127 *$ & Acute knees pain & $0.094 *$ \\
\hline Acute ankles pain & $0.120 *$ & Acute ankles pain & $0.087 * *$ \\
\hline
\end{tabular}

${ }^{*}$ Correlation is significant at the 0.05 level (2 tailed)

${ }^{* *}$ Correlation is significant at the 0.001 level ( 2 tailed) 
Using logistic regression model we found that increased body height protect acute lower pain among schoolchildren with normal BMI $(\beta=-0.089,95 \% \mathrm{CI},-9.730--0.023, \mathrm{P}<0.049)$; and protect acute upper back pain $(\beta=-0.356$, 95\%CI, -14.077- -3.878, $\mathrm{P}<0.001)$ and chronic lower back pain $(\beta=-0.356,95 \% \mathrm{CI},-14.077--3.878, \mathrm{P}<0.001)$ among obese schoolchildren (as shown in Table 4).

Table 4 Logistic regression model assessing association between body height (dependent variable) and acute and chronic musculosceletal pain in different part of the body (independent variables) among all respondents ( $\mathrm{n}=1315$ ) selecting step by step only in one of four groups of BMI classification groups

\begin{tabular}{|c|c|c|c|}
\hline $\begin{array}{l}\text { Body height and MSP association among underweight group of } \\
\text { respondents (selecting cases, } n=64 \text { ) }\end{array}$ & $\beta^{*}$ & $95 \% \mathrm{CI} * *$ & $\begin{array}{c}\text { P- } \\
\text { value }\end{array}$ \\
\hline Chronic neck pain & 0.037 & $-7.363-9.380$ & 0.810 \\
\hline Acute neck pain & -0.021 & $-9.003-7.908$ & 0.897 \\
\hline Chronic right shoulder pain & 0.234 & $-3.316-14.524$ & 0.213 \\
\hline Acute right shoulder pain & 0.198 & $-14.690-34.456$ & 0.423 \\
\hline Chronic right wrist pain & -0.063 & $-34.694-27.094$ & 0.806 \\
\hline Acute right wrist pain & -0.015 & $-13.858-12.911$ & 0.944 \\
\hline Chronic upper back pain & 0.096 & $-9.615-15.723$ & 0.630 \\
\hline Acute upper back pain & -0.119 & $-11.331-4.905$ & 0.430 \\
\hline Chronic lower back pain & -0.448 & $-50.020-10.853$ & 0.202 \\
\hline Acute lower back pain & 0.479 & $-4.992-63.242$ & 0.093 \\
\hline Chronic hips pain & 0.412 & $-9.682-50.927$ & 0.178 \\
\hline Acute hips pain & 0.212 & $-29.678-8.468$ & 0.269 \\
\hline Chronic knees pain & 0.208 & $-4.744-22.909$ & 0.193 \\
\hline Acute knees pain & 0.046 & $-2.143-6.627$ & 0.316 \\
\hline $\begin{array}{l}\text { Body height and MSP association among normal weight group } \\
\text { of respondents (selecting cases, } n=842 \text { ) }\end{array}$ & $\beta^{*}$ & $95 \% \mathrm{CI}$ & $\begin{array}{l}\text { P- } \\
\text { value }\end{array}$ \\
\hline Chronic neck pain & 0.010 & $-1.896-2.478$ & 0.794 \\
\hline Acute neck pain & 0.023 & $-1.665-2.963$ & 0.582 \\
\hline Chronic right shoulder pain & 0.033 & $-2.095-6.026$ & 0.342 \\
\hline Acute right shoulder pain & 0.066 & $-0.345-3.828$ & 0.102 \\
\hline Chronic right wrist pain & 0.066 & $-1.386-10.639$ & 0.131 \\
\hline
\end{tabular}




\begin{tabular}{|c|c|c|c|}
\hline Acute right wrist pain & -0.061 & $-11.072-1.843$ & 0.161 \\
\hline Chronic upper back pain & 0.044 & $-1.413-4.200$ & 0.330 \\
\hline Acute upper back pain & 0.018 & $-2.509-3.712$ & 0.704 \\
\hline Chronic lower back pain & 0.014 & $-1.759-2.537$ & 0.722 \\
\hline Acute lower back pain & -0.089 & $-9.730--0.023$ & 0.049 \\
\hline Chronic hips pain & 0.086 & $-0.247-10.168$ & 0.062 \\
\hline Acute hips pain & 0.042 & $-2.342-6.052$ & 0.386 \\
\hline Chronic knees pain & 0.046 & $-2.143-6.627$ & 0.316 \\
\hline Acute knees pain & 0.027 & $-2.514-4.890$ & 0.529 \\
\hline $\begin{array}{l}\text { Body height and MSP among over weight group of respondents } \\
\text { (selecting cases, } n=243 \text { ) }\end{array}$ & $\beta^{*}$ & $\begin{array}{l}\text { 95\% Confidence } \\
\text { interval }\end{array}$ & $\begin{array}{c}\text { P- } \\
\text { value }\end{array}$ \\
\hline Chronic neck pain & -0.027 & $-4.771-3.345$ & 0.729 \\
\hline Acute neck pain & 0.130 & $1.152-8.416$ & 0.136 \\
\hline Chronic right shoulder pain & 0.080 & $-3.391-12.076$ & 0.253 \\
\hline Acute right shoulder pain & 0.060 & $-3.109-6.586$ & 0.480 \\
\hline Chronic right wrist pain & -0.004 & $-16.242-15.263$ & 0.951 \\
\hline Acute right wrist pain & 0.057 & $-6.232-13.928$ & 0.453 \\
\hline Chronic upper back pain & -0.077 & $-9.365-3.479$ & 0.368 \\
\hline Acute upper back pain & -0.057 & $-9.402-4.937$ & 0.540 \\
\hline Chronic lower back pain & -0.045 & $-6.606-3.357$ & 0.572 \\
\hline Acute lower back pain & 0.154 & $0.511-21.364$ & 0.062 \\
\hline Chronic hips pain & -0.032 & $-13.763-9.798$ & 0.741 \\
\hline Acute hips pain & 0.089 & $-3.493-10.644$ & 0.320 \\
\hline Chronic knees pain & 0.075 & $-11.349-4.856$ & 0.431 \\
\hline Acute knees pain & 0.083 & $-3.980-10.644$ & 0.365 \\
\hline $\begin{array}{l}\text { Body height and MSP association among obese weight group } \\
\text { of respondents (selecting cases, } n=166 \text { ) }\end{array}$ & $\beta^{*}$ & 95\%CI & $\begin{array}{c}\text { P- } \\
\text { value }\end{array}$ \\
\hline Chronic neck pain & -0.086 & $-7.271-2.773$ & 0.378 \\
\hline Acute neck pain & 0.119 & $-2.440-9.403$ & 0.247 \\
\hline Chronic right shoulder pain & 0.117 & $-2.170-15.438$ & 0.139 \\
\hline Acute right shoulder pain & -0.108 & $-10.916-3.814$ & 0.342 \\
\hline Chronic right wrist pain & 0.051 & $-13.011-0.044$ & 0.621 \\
\hline
\end{tabular}




\begin{tabular}{|l|l|l|l|}
\hline Acute right wrist pain & 0.132 & $-2.917-10.624$ & 0.263 \\
\hline Chronic upper back pain & 0.200 & $-1.548-15.063$ & 0.110 \\
\hline Acute upper back pain & -0.356 & $-14.077--3.878$ & $\mathbf{0 . 0 0 1}$ \\
\hline Chronic lower back pain & -0.356 & $-14.077--3.878$ & $\mathbf{0 . 0 0 1}$ \\
\hline Acute lower back pain & -0.252 & $-34.641-10.342$ & 0.288 \\
\hline Chronic hips pain & 0.029 & $-22.494-25.474$ & 0.902 \\
\hline Acute hips pain & 0.267 & $-2.175-23.934$ & 0.102 \\
\hline Chronic knees pain & -0.076 & $-18.465-11.542$ & 0.649 \\
\hline Acute knees pain & 0.095 & $-6.668-15.867$ & 0.421 \\
\hline
\end{tabular}

$* \boldsymbol{\beta}$, Beta coefficient in regression ANOVA analysis of potential predictors of musculosceletal pain

$* * 95 \%$ CI, 95\% Confidence interval

\section{Discussion}

This school-based survey adds important knowledge about 1315 Bosnia and Herzegovina schoolchildren $11.31 \pm 1.48$ years old, $146.18 \pm 113.50 \mathrm{~cm}$ tall, $60.89 \pm 30.83$ hard, who most often self-reported neck pain, right shoulder pain and lower back pain as musculosceletal trouble in the last 12 months (chronic pain). Forty eight percent of this entire population-based cohort reported daily current MSP, the most frequent acute neck pain.

Overweight and obese children report more musculoskeletal problems in daily life than their normal-weight peers [4-6, 15-16]. Furthermore, they reported more ankle and foot problems than did the children who were of normal weight $(\mathrm{OR}=1.92 ; 95 \% \mathrm{CI}, 1.15-3.20)$. Overweight and obese children aged 12 to 17 years consulted their family physicians more often with lower extremity problems than did the normal-weight children $(\mathrm{OR}=1.92 ; 95 \% \mathrm{CI}$, 1.05-3.51) [6]. Our school children with normal weight have suffered more often acute neck pain, acute right shoulder pain, acute upper back pain, chronic upper back pain, acute low back pain and chronic low back pain than those with overweight or obesity (regarding BMI categories). Finally, overweight and obesity has not been associated with MSP in school children. It is hypothetically assumed that MSP in children with normal weight is actually associated with progressive growth of children at this age. Adolescence plays an important role on the natural evolution of MSP since during the pre-adolescence period MSP tends to resolve. Conversely, adolescents continue suffering from MSP to a greater extent than the pre-pubertal ones [17-20].

Possible limitation of the present study was the fact that it was based on a survey through a self- rated questionnaire. As the study was cross- sectional in design, it is difficult to determine the temporal associations between the factors.

Our results clearly show protective role of body height $(\mathrm{cm})$ in normal weight children regarding acute lower back pain $(\beta=-0.089,95 \% \mathrm{CI},-9.730--0.023, \mathrm{P}<0.049)$, and in obese school children regarding acute upper back pain $(\beta=-0.356,95 \% \mathrm{CI},-14.077--3.878, \mathrm{P}<0.001)$ and chronic low back pain $(\beta=-0.356,95 \% \mathrm{CI},-14.077--3.878$, 
$\mathrm{P}<0.001)$. Meanwhile, body weight $(\mathrm{kg})$ was not associated with MSP in step by step selected different BMI categories during regression analysis.

The main findings of this study were that those elevated body mass indexes have been not independent predictor of MSP in different part of body among schoolchildren. On other site, growth in height during adolescence has rarely been examined in relation to growing MSP.

Conclusion School children with normal weight have suffered more often pain than those with overweight or obesity. Body height $(\mathrm{cm})$ showed a protective role in normal weight children regarding acute lower back pain and in obese schoolchildren regarding acute upper back pain and chronic lower back pain. Factors for MSP development should be explored especially in the population of children and adolescents. Due to height growth, body height plays a significant role in the protection of musculoskeletal trouble [20]. Risk factors for MSP are not always comparable with risk factors for MSP of adults.

Acknowledgement: None. No funding to declare.

Conflict of interest: The authors declare no conflict of interest.

\section{References}

1. El-Metwally A, Salminen JJ, Auvinen A, Macfarlane G, Mikkelsson M. Risk factors for development of nonspecific musculoskeletal pain in preteens and early adolescents: a prospective 1-year follow-up study. BMC Musculoskelet Disord. 2007; 8:46. DOI: 10.1186/1471-2474-8-46. Available online (accessed on 23. May 2007).

2. O’Sullivan P, Beales D, Jensen L, Murray K, Myers T. Characteristics of chronic non-specific musculoskeletal pain in children and adolescents attending a rheumatology outpatients clinic: a cross-sectional study. Pediatr Rheumatol. 2011; 9:3. DOI: 10.1186/1546-0096-9-3. Available online (accessed on 19. Jan 2011).

3. Krul M, van der Wouden JC, Schellevis FG, Suijlekom- Smit LW, Koes BW. Musculoskeletal Problems in Overweight and Obese Children. Ann Fam Med. 2009; 7(4):352-6. DOI: 10.1370/afm.1005. Available online (accessed on 1. July 2009).

4. Shiri R, Karppinen J, Leino-Arjas P, Solovieva S, Viikari-Juntura E. The association between obesity and low back pain: a meta-analysis. Am J Epidemiol. 2009; 171 (2):135-54. DOI: 10.1093/aje/kwp356. Available online (accessed on 11. Dec 2009).

5. Ivar T, Nilsen L, Holtermann A, Mork PJ. Physical exercise, body mass index, and risk of chronic pain in the low back and neck/shoulders: longitudinal data from the Nord-Trondelag health study. Am J Epidemiol. 2011; 174(3): 267-73. DOI:10.1093/aje/kwr087. Available online (accessed on 1. Aug 2011). 
6. Cardon G, Balagué F. Acute low back in high school adolescents in Southern Brazil: Prevalence and associated factors. European Spine Journal. 2012; 21(7):1234-40. DOI: 10.1007/s00586-011-2056-3. Available online (accessed on 3. Nov 2011).

7. Hershkovich O, Friedlander A, Gordon B, Arzi H, Derazne E, Tzur D, Shamis A, Afek A. Association between body mass index, body height, and prevalence of spine deformities. Spine J. 2014; 14(8):1581-7. DOI: 10.1016/j.spinee.2013.09.034. Available online (accessed on 19. Oct 2013).

8. Jespersen E, Rexen CT, Franz C, Møller NC, Froberg K, Wedderkopp N. Musculoskeletal extremity injuries in a cohort of schoolchildren aged 6-12: A 2.5-year prospective study. Scand J Med Sci Sports. 2015; 25(2):251-8. DOI. 10.1111/sms.12177. Available online (accessed on 29. Jan 2014).

9. Kamper SJ, Henschke N, Hestbaek L, Dunn KM,Williams CM. Musculoskeletal pain in children and adolescents. Braz J Phys Ther. 2016; 20(3):275-84. DOI:10.1590/bjpt-rbf.2014.0149. Available online (accessed on 16. Feb 2016).

10. Lardon A, Leboeuf-Yde C, Le Scanff C, Wedderkopp N. Is puberty a risk for back pain in the young? A systematic critical literature review. Chiropr Man Therap. 2014; 22-7. DOI: 10.1186/s12998-014-0027-6. Available online (accessed on 15. Oct 2014).

11. Friedrichsdorf SJ, Giordano J, Deasai Dakoji K, Warmuth A, Daughtry C, Schulz CA (2016) Chronic Pain in Children and Adolescents: Diagnosis and Treatment of Primary Pain Disorders in Head, Abdomen, Muscles and Joints. Children (Basel). 2016: 3 (4):42. DOI: 10.3390/children3040042. Available online (accessed on 10. Dec 2016).

12. Kuorinka I. Jonsson B, Kilbom A, Vitenberg H, Biering- Sørensen F, Andersson G, Jørgensen K. Standardized Nordic questionnaire for the analysis of musculosceletal symptoms. Appl Ergon. 1987; 18:2337.

13. Crawford JO. The Nordic Musculoskeletal Questionnaire. Occup Med. 2007; 57(4): 300-1. DOI: 10.1093/occmed/kqm036. Available online (accessed on 1. June 2007).

14. CDC Centres for Disease Control and Prevention. Clinical Growth Charts. Homepage on the Internet. Clinical Growth Charts. Available online: URL (accessed 18 March 2018).

15. Viester L,Verhagen EALM, Oude Hengel KM, Koppes LLJ, van der Beek AJ, Bongers PM. The relation between body mass index and musculoskeletal symptoms in the working population. BMC Musculoskelet Disord. 2013; 14:238. DOI: 10.1186/1471-2474-14-238. Available online (accessed on 12. Aug 2013).

16. Smith SM, Sumar B, Dixon KA. Musculosceletal pain in overweight and obese children. Int J Obes (Lond). 2014; 38(1):11-5. DOI: 10.1038/ijo.2013.187. Available online (accessed on 29. Oct 2013). 
17. Speretto F, Brachi S, Vittadello F, Zulian F. Musculoskeletal pain in schoolchildren across puberty: a 3-year follow-up study. Pediatr Rheumatol. 2015; 13:16. DOI: 10.1186/s12969-015-0014-z. Available online (accessed on 15. May 2015).

18. Rhee H. Relationships between physical symptoms and pubertal development. $J$ Pediatr Health Care.2005;19(2): 95-103. DOI: 10.106/j.pedhc.2004.004. Available online (accessed on Mar-Apr 2005).

19. Eckhoff C, Straume B, Kvemmo S. Multisite musculoskeletal pain in adolescence and later mental health disorders: a population -based registry study of Norwegian youth: the NAAHS cohort study. BMJ Open. 2017; 7(2): e012035. DOI: 10.1136/bmjopen-2016-012035. Available online (accessed on 10. Feb 2017).

20. Hollander K, Elsabe de Viliers J, Sehner S, Wegscheider K, Braumann KM, Venter R, Zech A. Growing-up (habitually) barefoot influences the development of foot and arch morphology in children and adolescents. Sci Rep. 2017; 7: 8079. DOI: 10.1038/s41598-017-07868-4. Available online (accessed on 14 Aug 2017). 